OPEN ACCESS

Edited by:

Vida Vafaizadeh,

University of Basel, Switzerland

Reviewed by:

Masaru Miyano,

Lawrence Berkeley National Laboratory, United States

Yoo-Ah Kim,

National Institutes of Health $(\mathrm{NIH})$,

United States

*Correspondence:

Walid T. Khaled

wtk22@cam.ac.uk

Specialty section:

This article was submitted to

Molecular Medicine,

a section of the journal

Frontiers in Cell and Developmental

Biology

Received: 27 September 2019 Accepted: 27 November 2019 Published: 20 December 2019

Citation:

Waterhouse MP, Ugur $R$ and Khaled WT (2019) Therapeutic and Mechanistic Perspectives of Protein Complexes in Breast Cancer. Front. Cell Dev. Biol. 7:335 doi: 10.3389/fcell.2019.00335

\section{Therapeutic and Mechanistic Perspectives of Protein Complexes in Breast Cancer}

\author{
Mark P. Waterhouse, Rosie Ugur and Walid T. Khaled* \\ Department of Pharmacology, University of Cambridge, Cambridge, United Kingdom
}

Breast cancer affects one in eight women making it the most common cancer in the United Kingdom, accounting for $15 \%$ of all new cancer cases. One of the main challenges in treating breast cancer is the heterogeneous nature of the disease. At present, targeted therapies are available for hormone receptor- and HER2-positive tumors. However, no targeted therapies are currently available for patients with triple negative breast cancer (TNBC). This likely contributes to the poor prognostic outcome for TNBC patients. Consequently, there is a clear clinical need for the development of novel drugs that efficiently target TNBC. Extensive genomic and transcriptomic characterization of TNBC has in recent years identified a plethora of putative oncogenes. However, these driver oncogenes are often critical in other cell types and/or transcription factors making them very difficult to target directly. Therefore, other approaches may be required for developing novel therapeutics that fully exploit the specific functions of TNBC oncogenes in tumor cells. Here, we will argue that more research is needed to identify the protein-protein interactions of TNBC oncogenes as a means for (a) mechanistically understanding the biological function of these oncogenes in TNBC and (b) providing novel therapeutic targets that can be exploited for selectively inhibiting the oncogenic roles of TNBC oncogenes in cancer cells, whilst sparing normal healthy cells.

Keywords: transcription factor, breast cancer, TNBC, protein-protein interaction, protein complexes, cancer therapy, PROTAC, post-translational modification

\section{BREAST CANCER SUBTYPES AND ASSOCIATED THERAPIES}

Breast cancer is the most common cancer in the United Kingdom, accounting for 15\% of all new cancer cases, and is the leading cause of cancer death in women worldwide (Bray et al., 2018). Historically, breast cancers have been classified based on the expression of several cellsurface receptors, namely the estrogen receptor (ER), progesterone receptor (PR), and human epidermal growth factor receptor 2 (HER2) (Onitilo et al., 2009). Based on the presence or absence of these markers, breast cancers can be broadly stratified into luminal A/B, HER2+, or basal-like (triple-negative) subtypes. Specifically, luminal A/B breast cancers are characterized as hormone receptor positive (high expression of ER and/or PR); HER2+ breast cancers are characterized by amplification of HER2 (and can be ER+ and/or PR+); and triple negative breast cancers (TNBCs) are characterized as hormone receptor negative and HER2 negative (ER-/PR-/HER2-) (Onitilo et al., 2009). Receptor status continues to act as a critical assessment for all breast cancers, likely due to the quick, easy and cost-effective stratification of patients to determine suitability for 
targeted treatments. These include tamoxifen, an ER modulator, and trastuzumab (Herceptin), a monoclonal antibody targeting the HER2 receptor, which are first-line therapies for ER+ tumors in pre-menopausal women and HER2+ tumors, respectively. Due to the availability of effective treatment options, hormone receptor positive breast cancers generally have a better prognosis (Fallahpour et al., 2017). Prior to the advent of modern therapies, HER2+ patients had a worse prognosis. However, since the introduction of HER2-targeted therapies, such as trastuzumab, there has been a significant improvement in prognosis (Slamon et al., 2001). Conversely, TNBCs (i.e., negative for all three hormone receptors) still lack targeted treatments and continue to have a comparatively poor prognosis (Dent et al., 2007; Onitilo et al., 2009).

For tumors that are susceptible to targeted therapies various therapies are available (Lumachi et al., 2013; Fallahpour et al., 2017). For hormone receptor-positive cancers, these include selective ER modulators, inhibitors of the aromatase enzyme and antigonadotropic therapies. Patients with HER2+ tumors also benefit from the availability of the monoclonal antibodies trastuzumab and pertuzumab, which function by preventing HER2 from functioning by inhibiting HER2-associated signaling (Molina et al., 2001; Agus et al., 2002; Franklin et al., 2004; Junttila et al., 2009). For tumors that are unsuitable for targeted therapies (i.e., TNBC), treatment involves chemotherapy in combination with radiotherapy and/or surgery (Foulkes et al., 2010; Wahba and El-Hadaad, 2015). Chemotherapeutic agents that are currently approved for use in breast cancer therapy typically target DNA synthesis and repair pathways and tend to have more serious side effects. Mechanistically, these therapies comprise alkylating agents that irreversibly crosslink DNA and lead to apoptosis (Hall and Tilby, 1992) [cyclophosphamide, mitomycin C (Tomasz, 1995)]; inhibitors of DNA biosynthesis enzymes such as dihydrofolate reductase [methotrexate (Goodsell, 1999)], thymidine synthase [fluorouracil (Longley et al., 2003)] and type II topoisomerase [mitoxantrone (Fox, 2004)]; or DNA intercalators that inhibit DNA and RNA synthesis [epirubicin, doxorubicin (Gewirtz, 1999)] (Figure 1A). Cytoskeletal drugs are also approved for use in breast cancer therapy (e.g., paclitaxel) and block cell cycle progression by stabilizing microtubule polymers (Horwitz, 1994; Figure 1B).

In hormone receptor and/or HER2+ positive tumors, it appears that much efficacy is derived from the availability of therapies targeting protein-protein interactions (PPIs) that drive disease. These therapies, which include tamoxifen, anastrozole and trastuzumab, either directly or indirectly block interactions between growth factors and their receptors. However, due to the lack of actionable receptors in TNBC, chemotherapy remains the first-line standard of care in combination with radiotherapy and/or surgery. It is apparent that these non-targeted chemotherapeutic agents represent generic therapeutic strategies that broadly target cancerous tissues, as they preferentially target rapidly dividing cells such as those found in tumors. However, normal cells that divide rapidly such as those in the digestive tract, hair follicles, and bone marrow are also highly susceptible to cytotoxicity, which leads to common chemotherapeutic side effects such as mucositis, alopecia, and myelosuppression with subsequent immunosuppression (Partridge et al., 2001). As a result, there is a clear clinical need for the identification of actionable targets in TNBC that can be used as the basis for the production of new and more targeted therapies. It is likely that, to ensure specificity in targeting, the identification of PPIs or protein networks that drive disease will be necessary for this purpose.

A promising example of this concept is the inhibition of interactions that induce immune tolerance such as the PD-1/PDL1 axis (Gatalica et al., 2014), inhibition of which promotes T-cell proliferation, survival and cytotoxicity (Figure 1A). Most recently this has included the approval of atezolizumab, an anti-PD-L1 antibody, in combination with chemotherapy for the treatment of PD-L1+ metastatic TNBC by the US FDA in March 2019 (Schmid et al., 2018; Dolgin, 2019). Another example is the recent use of poly ADP ribose polymerase (PARP) inhibitors for the treatment of BRCA1-mutated tumors. Although there continues to be a lack of targeted treatment options for TNBC patients, $\sim 15 \%$ of TNBC tumors are driven by germline mutations within the BRCA1 and BRCA2 genes (Engel et al., 2018). These mutations result in defective doublestrand DNA repair machinery and lead to the accumulation of DNA damage. PARP is another DNA repair protein that is crucial for the repair of single-strand DNA breaks (Audebert et al., 2004; Heale et al., 2006), which can develop into doublestrand breaks (DSBs) if not repaired before the initiation of DNA replication (Farmer et al., 2005). In this context, BRCA1/BRCA2 mutated tumors cannot repair these DSBs, ultimately resulting in cell death, whereas normal cells can compensate for the loss of PARP function (Farmer et al., 2005). As a result, patients with mutated BRCA1/BRCA2 are suitable candidates for additional treatment with PARP inhibitors, such as the recently approved drug olaparib which was approved in 2019 in Europe for germline BRCA1/2-mutated HER2- breast cancer (Griguolo et al., 2018; Le and Gelmon, 2018). However, this therapy class is only suitable for patients with $B R C A$-mutated tumors and there is still intense interest in the identification of the molecular drivers of TNBC.

\section{MOLECULAR PROFILING OF BREAST CANCERS FOR TARGET IDENTIFICATION}

Much effort has been invested into the molecular profiling of breast cancers for the identification of novel drivers in TNBC pathogenesis and to better define breast cancer subtypes. The first of these classification models, proposed by Sørlie et al. (2003), was based on the transcriptomic profiling of 115 malignant breast tumors and identified five intrinsic subtypes of breast cancers (Sørlie et al., 2001, 2003). Although the identification of these intrinsic subtypes has provided much insight into breast cancer biology, attempts to define possible somatic drivers of breast cancer subtypes has remained difficult due to the heterogeneity of the disease as well as a lack of clear driver mutations. More recent work has aimed to tackle this issue by integrating genomic and transcriptomic breast cancer data in much larger patient sizes, a prime example of which is the recent METABRIC 

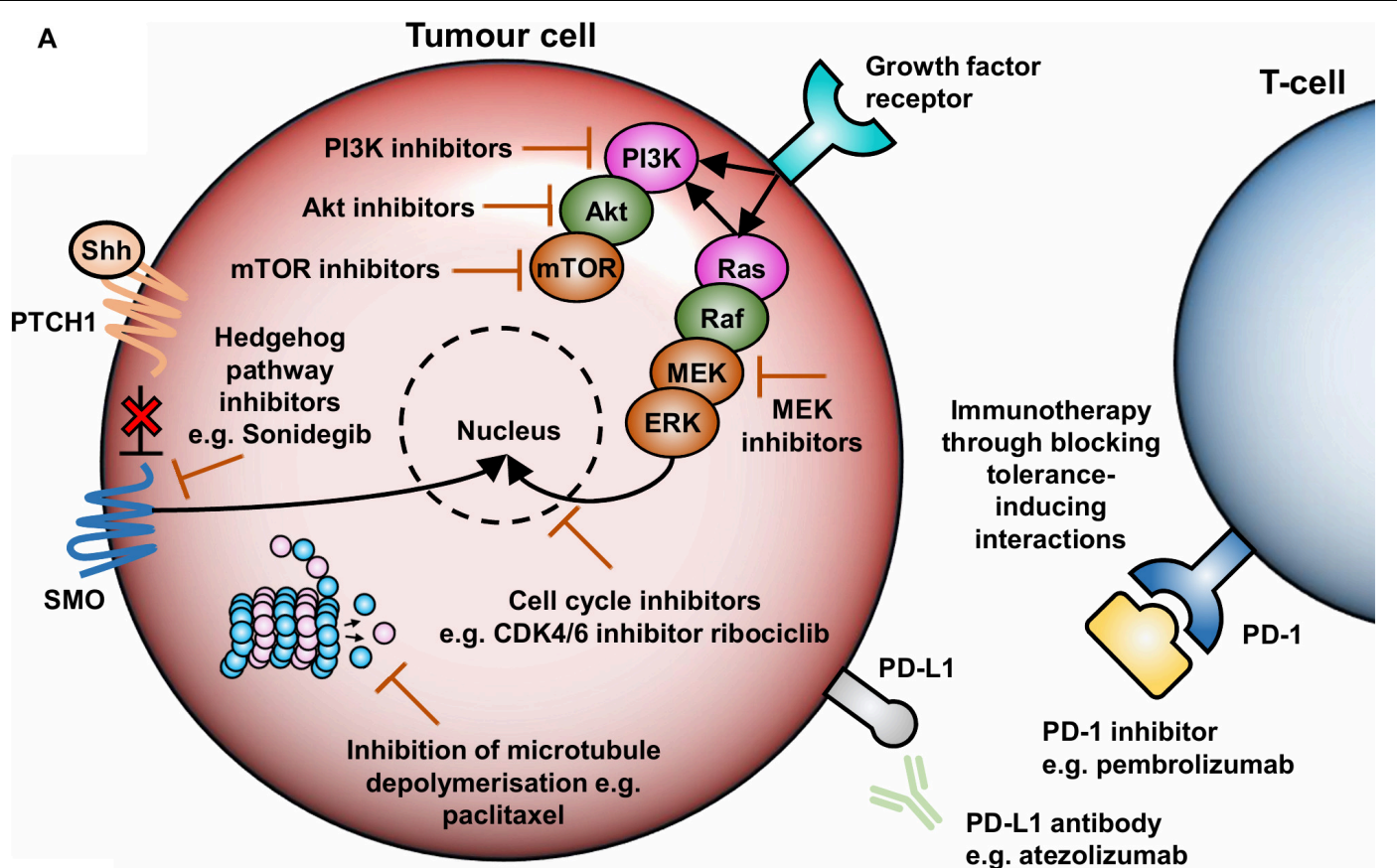

PD-1 inhibitor

e.g. pembrolizumab

PD-L1 antibody

e.g. atezolizumab

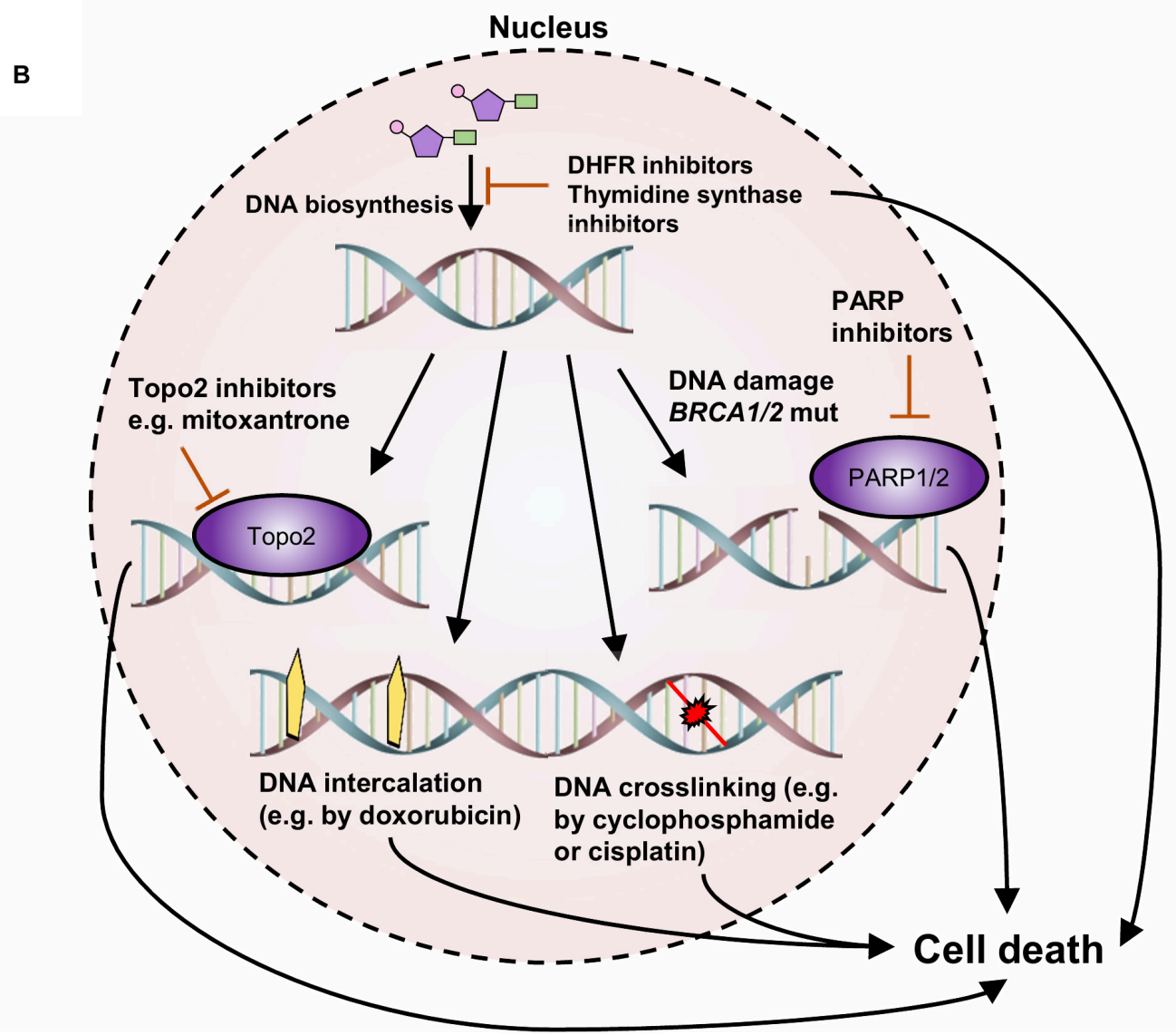

FIGURE 1 | An overview of current and emerging agents for TNBC therapy. (A) TNBC therapies targeting the cell surface and cytoplasm. Cell surface therapies include inhibitors of immune tolerance inducing proteins such as PD1 and PD-L1. Cytoplasmic therapies include inhibitors of the Ras/MAPK pathway, especially MEK inhibitors, inhibitors of the PI3K/AKT/mTOR pathway, inhibitors of the Hedgehog signaling pathway, and cell cycle inhibitors such as paclitaxel and CDK inhibitors. (B) TNBC therapies targeting the nucleus. These therapies tend to target DNA synthesis and repair pathways or affect DNA viability to induce cell-cycle arrest and cell death. 
dataset. This work characterized the genomic and transcriptomic architecture of 2000 breast tumors (Curtis et al., 2012) and resulted in the identification of 10 novel molecular subgroups, known as integrative clusters, which are clustered according to copy number alterations and gene expression data (Dawson et al., 2013). Crucially, each integrative cluster is associated with distinct clinical features and outcomes (Dawson et al., 2013). In addition, the clusters have identified heterogeneity within tumors classified according to receptor status and divided all previously identified intrinsic subtypes into separate groups. Additional transcriptomic studies have further highlighted the heterogeneity of TNBC, which include studies by Lehmann et al. $(2011,2016)$ and Burstein et al. (2015), both of which identified four molecular subtypes of TNBC. As a result, breast cancer classification is now evolving to describe a number of distinct molecular subgroups based on multiple genomic factors, which has produced more robust patient classifiers and is leading to a new stratification and treatment paradigm for breast cancer patients. However, despite this progress in the molecular characterization of TNBC, these tumors remain to be mostly characterized by TP53 alterations and copy number alterations involving $5 \mathrm{q}$ loss and gains at $8 \mathrm{q}$, 10p and 12p (Dawson et al., 2013).

A limited number of studies have therefore attempted to investigate the mutational landscape in TNBC, which has mostly identified that TNBC is characterized by a low rate of activating point mutations in common oncogenes, as well as extensive individually rare mutations in other genes (Shi et al., 2018). However, TNBCs appear to be particularly enriched for alterations in tumor suppressor proteins, such as TP53, RB1, and $P T E N$, as well as oncogenic alterations in the PI3K/AKT pathway (Curtis et al., 2012; Koboldt et al., 2012). Regardless, common TNBC “oncogenes” such as PIK3CA and other actionable targets, such as the Ras/MAPK (Balko et al., 2013), JAK/STAT (Marotta et al., 2011), Wnt (DiMeo et al., 2009), TGF- $\beta$ (Bhola et al., 2013), Hedgehog (Liu et al., 2006), and Notch (Harrison et al., 2010) pathways, are all critical genes/signaling pathways in a wide range cell types and contexts. As a result, any therapies designed against these pathways are highly likely to result in off-target cytotoxicity. Overall therefore, genome-wide studies have failed to identify driving mutations distinct from those affecting TP53, PIK3CA, and PTEN (Peluffo et al., 2019), and new therapeutic angles are required to define better and more specific targets for the production of TNBC therapies. One such angle to consider is that alterations in epigenetics and transcriptional machinery may be largely contributing to the transcriptional dysregulation seen in TNBC malignancies.

\section{TRANSCRIPTION FACTOR TARGETING FOR POTENTIAL ENHANCED THERAPEUTIC SPECIFICITY}

Downstream effectors of traditionally targeted pathways, namely transcription factors (TFs) involved in normal cellular function, are often those subjected to dysregulation resulting in cancer (Bass et al., 2009). Indeed, many cancer-related events either directly involve TFs or indirectly modulate TF activity. This highlights targeting TFs as a promising anticancer strategy and as potentially superior therapeutic targets compared to upstream signaling proteins and kinases (Konstantinopoulos and Papavassiliou, 2011). Our progression in understanding of the mechanistic properties of TFs and their associated networks, in both diseased and normal cells, has created huge potential for precision medicine in cancer. For example, targeting oncogenic TFs may lead to preferential cancer cell death in tumors that display TF dependency, whereas normal cells may be more likely to tolerate a loss of TF function due to redundancies in normal signaling pathways. One such case is the TRPS1 TF, which demonstrates breast lineage-specific transcriptional dependency, likely due to lineage-restricted expression (Witwicki et al., 2018). As a result, breast cancer cells lines display sensitivity to TRPS1 shRNA targeting compared to cell lines derived from colon, neuroblastoma, leukemia, prostate, and rhabdoid tumors (Witwicki et al., 2018). TFs in this context are therefore likely to have a high therapeutic potential, owing to their critical role in tumor pathogenesis along with their dispensability for physiologic cell function. Accordingly, many studies have tried to capture the transcriptional landscape of TNBC, thus identifying highly expressed genes and TFs that may be liable to therapeutic targeting. However, TFs have long been considered "undruggable" targets, which may result from the large interaction surface areas used by TFs for protein-DNA and PPIs as well as their predominant nuclear localization, which makes them less accessible to therapeutic agents (Yan and Higgins, 2013).

Despite these challenges, there are various opportunities available for targeting TFs at different functional levels. For example, TFs may be directly or indirectly targeted through inhibition (or activation) at the expression level, at the PPI level, at the post-translational modification level, at the protein/DNA binding level, through the binding of a small molecule in an inhibition/activation pocket or through physical degradation (Figure 2). In addition, post-translational modifications, which may result in context-specific PPIs and/or differential assembly of epigenetic remodeling complexes, must also be considered. To date, over 450 unique protein modifications have been described, including phosphorylation, acetylation, ubiquitination, methylation, and SUMOylation, which can alter target protein activity, intracellular distribution, protein interactions and protein longevity (Venne et al., 2014). For phosphorylation alone, there are over 500 different kinases in mammals (Woolfrey and Dell'Acqua, 2015), some of which could conceivably be expressed in a tissue-specific manner and may therefore give rise to differing versions of the same proteins in various tissues.

Like all other cellular proteins, TF expression is controlled by transcriptional activators and repressors (such as other TFs or itself in a feedback loop) as well as by epigenetic machinery. Aberrant activity of these processes may therefore result in oncogenic transcriptional programs. For example, oncogenic gene translocation and consequent juxtaposition of the c-MYC gene with enhancer elements has been reported in multiple myeloma, which may enhance c-MYC expression (Shou et al., 2000), and aberrant expression of the HOXA cluster of TFs 


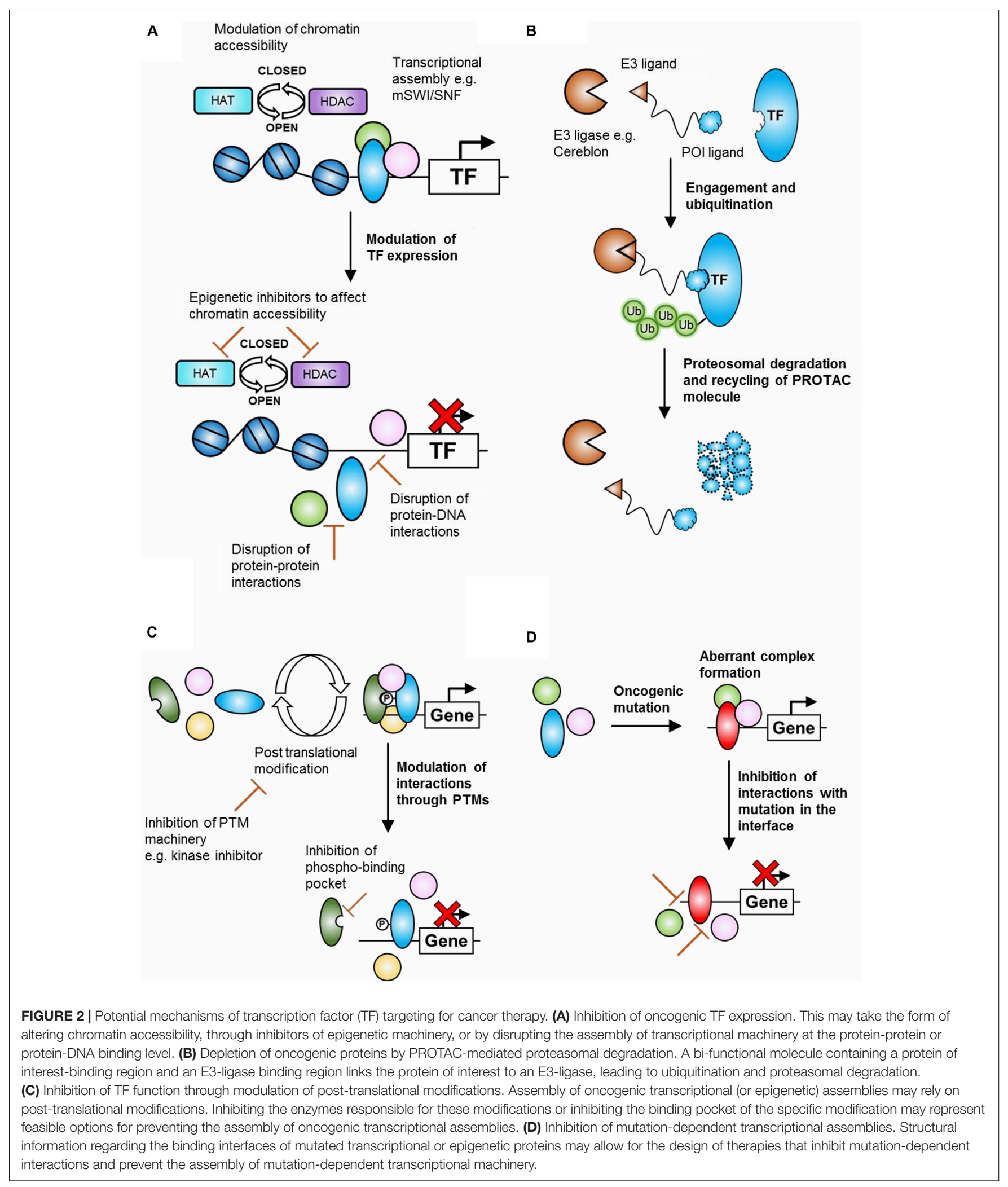

has been reported in several aggressive acute leukemias as a result of oncogenic rearrangements of the MLL1 gene, a histone methyltransferase (Kawagoe et al., 1999; Guenther et al., 2005).
More specifically, rearrangements of the $M L L 1$ gene can lead to the production of over 70 in-frame oncogenic fusion proteins, which can add functionality by enabling interactions with histone 
methyltrasferases such as DOT1L (Krivtsov et al., 2007) or may direct the MLL complex to unintended genomic areas, resulting in an aberrant transcriptional program. Therapies have been designed for both of these contexts, typically through regulation at the epigenetic level. For example, HDAC and histone methyltransferase inhibitors (e.g., against DOT1L), both of which associate with the MLL complex, have entered clinical trials for acute myeloid leukemia (Daigle et al., 2011; Chen et al., 2013; Fredly et al., 2013; Morabito et al., 2016), whereas negative regulation of oncogenic c-MYC has been achieved, for example, by inhibitors of BRD4 (such as JQ1) which displace BRD4 from the c-MYC promoter (Fowler et al., 2014). Control of HOXA expression has also been attempted through disruption of the MLL complex, for example by inhibiting the incorporation of WDR5 into the MLL complex which is required for the enzymatic activity of MLL1 (Li et al., 2016; Karatas et al., 2017). These examples represent indirect TF targeting at the epigenetic and PPI levels.

As well as indirect inhibition of TF function, direct inhibition of TF interactions may be an attractive therapeutic approach. Targets in this case may include single TF homodimers, a specific heterodimeric TF pair or a multimeric transcriptional complex. Indeed, TFs have the potential to form a large number of dimeric structures with distinct biological properties (over 500 dimers in human and up to 2500 dimers when considering alternate splicing) that can allow for elaborate fine-tuning of responses (Amoutzias et al., 2008). The concentration of each monomer in the cell, its post-translational modifications and its binding affinity for other monomers all play a role in determining dimer formation and, consequently, will determine which signaling process will dominate. These regulatory mechanisms therefore offer multiple levels of complexity and likely represent an underexploited therapeutic opportunity, as targeting specific TF dimers or specifically modified TFs (e.g., phosphorylated at a specific position) may offer exquisite therapeutic specificity. This may become a viable approach through the identification of TF states that contribute toward disease pathogenesis, especially as TFs have traditionally been considered undruggable.

A good example of this concept is the Myc-Max and Mad-Max heterodimerization system, whereby Max is a ubiquitous protein that can heterodimerize with either Myc or Mad (Grandori et al., 2000; Lüscher, 2001). Similarly, Myc and Mad can only heterodimerize with Max, but not each other. Upon formation of the Myc-Max heterodimer, recruitment of the mSWI/SNF nucleosome remodeling complex or histone acetyltransferases (HATs) occurs at the promoters of target genes, resulting in transcriptional activation. Conversely, formation of the MaxMad heterodimer leads to recruitment of HDACs, which results in silencing of target genes and is antagonistic to the MycMax heterodimer. As a result, variations in the concentrations or affinity of these complexes can lead to a transcriptional bias and potentially alter the oncogenic capacity of the cell. As previously discussed, post-translational modifications may offer an additional level of complexity and can determine the transition to a functional dimeric TF pair. This has been observed through the phosphorylation of STAT proteins (Levy and Darnell, 2002) as well as ReIB, which leads to the formation of p100-ReIB dimers
(Maier et al., 2003). Another example is the phosphorylation of the bHLH protein E47, which blocks formation of the homodimer and favors formation of a heterodimer with MyoD, leading to the activation of muscle-specific transcriptional activity (Lluís et al., 2005). It is therefore feasible to suggest that phosphorylated versions of TFs that participate in oncogenic interactions or transcriptional programs may represent attractive therapeutic targets in the future, especially if the phosphorylated protein does not exist or is very rare in healthy tissues.

\section{TARGETING PROTEIN NETWORKS AND CHROMATIN RE-MODELERS}

In addition to TFs, it is important to consider the role played by chromatin modulators in driving transformation. Mutated protein members "hijack" remodeling machinery to localize in different areas of the genome, leading to aberrant gene expression. Although TFs in cancer are undoubtedly important, open chromatin is more likely to facilitate gene expression. Therefore, the targeting of PPIs specific to oncogenically activated chromatin modulators may offer a more viable method to silence dysregulated transcription in cancer. One clear example is the BAF or $\mathrm{mSWI} / \mathrm{SNF}$ complex, where genes encoding subunits or associated proteins are mutated in over $20 \%$ of cancers (Pierre and Kadoch, 2017). Such a high frequency of mutations correlated with specific oncogenic phenotypes can be attributed to a high degree of genetic non-redundancy within the complex. An example is SMARCB1 inactivation in early pediatric rhabdoid tumors, which is considered the sole genetic driving event in an otherwise genomically stable malignancy (Wang et al., 2017). Such stability is indicative of epigenetic changes caused by an oncogenically activated BAF complex. The loss of SMARCB1 reduces levels of the BAF complex, impairing normal function and transcriptional homeostasis. Subsequent alteration in genome-wide targeting reduces BAF binding to typical enhancers required for transcription of cell differentiation genes. Instead, remaining SMARCB1-deficient complexes maintain binding at super enhancers, causing preferential transcription of genes required for current cell identity maintenance, which may be due to higher affinity BAF complex binding at these sites. When specific proliferative progenitor cells are affected, cells are effectively locked into a highly proliferative and lowly differentiated state due to impaired enhancer targeting working to drive oncogenic transformation (Wang et al., 2017). Expression of another subunit of the complex, ARID1A, is lost in colon cancer in mice causing a similar reduction in levels of the BAF complex (Mathur et al., 2017). This causes its absence at thousands of enhancers and subsequent reduction/change in gene expression. ARID1B has a similar binding preference and can compensate to some extent by binding in the place of ARID1A, but the presence of this altered complex causes extensive dysregulation of gene expression. This further highlights the importance of complex composition and the non-redundant nature of PPIs within this complex. There are therefore a great many potential targets for therapy within the BAF complex. Indeed, comprehensive understanding of 
the relationships between biochemistry and function must be reached in order to unlock their greatest potential.

Another emerging field for PPI targeting is the modulation of the ubiquitin pathway. For example, proteasome-mediated degradation can be biased toward the preferential break down of tumor suppressors and the apparent preservation of oncoproteins in cancer cells (Wertz and Wang, 2019). As the process is a cascade, there are several proteins which offer valuable targets for anticancer therapies. There are three classes of enzymes responsible for ubiquitination, E1, E2, and E3, which comprise 2, 40, and over 600 isozymes in humans respectively ( $\mathrm{Li}$ et al., 2008; Deshaies and Joazeiro, 2009; Schulman and Wade Harper, 2009). Although it has been possible to modulate the E1 and E2 members of the ubiquitination pathway, E3 enzymes have higher substrate specificity and offer greater potential for specific targeting. One of the most notable E3 PPIs for targeting is the MDM2:p53 interaction, where MDM2 is the negative regulator for p53 and therefore an important oncoprotein. Another method of modulation involving target proteins and E3 enzymes are Proteolysis targeting chimeras (PROTACs) (Sakamoto et al., 2001). PROTACs contain two moieties which independently bind a relevant target protein and an E3 ubiquitin ligase (Figure 2B). This brings the target into close proximity for ubiquitination by the E3 enzyme and marks the protein for degradation by the proteasome. This system has the advantage of being able to target proteins such as TFs, as PROTACs require only transient drug-target binding, whilst not inhibiting substrate activity. The ubiquitin pathway therefore offers an attractive therapeutic angle to the targeting of TFs, helping to modulate proteins which are otherwise difficult to mark.

It is clear that the wealth of existing proteomics data needs to be harnessed to address this area, looking at the specific interactions and phosphorylation states of putative TNBC oncogenes in disease contexts versus those observed in healthy tissue. This may take the form of targeted approaches using emerging techniques such as rapid immunoprecipitation mass spectrometry of endogenous proteins

\section{REFERENCES}

Agus, D. B., Akita, R. W., Fox, W. D., Lewis, G. D., Higgins, B., Pisacane, P. I., et al. (2002). Targeting ligand-activated ErbB2 signaling inhibits breast and prostate tumor growth. Cancer Cell 2, 127-137. doi: 10.1016/S1535-6108(02)00097-91

Amoutzias, G. D., Robertson, D. L., Van de Peer, Y., and Oliver, S. G. (2008). Choose your partners: dimerization in eukaryotic transcription factors. Trends Biochem. Sci. 33, 220-229. doi: 10.1016/j.tibs.2008.02.002

Audebert, M., Salles, B., and Calsou, P. (2004). Involvement of poly(ADP-ribose) polymerase-1 and XRCC1/DNA ligase III in an alternative route for DNA double-strand breaks rejoining. J. Biol. Chem. 279, 55117-55126. doi: 10.1074/ jbc.M404524200

Balko, J. M., Schwarz, L. J., Bhola, N. E., Kurupi, R., Owens, P., Miller, T. W., et al. (2013). Activation of MAPK pathways due to DUSP4 loss promotes cancer stem cell-like phenotypes in basal-like breast cancer. Cancer Res. 73, 6346-6358. doi: 10.1158/0008-5472.CAN-13-85

Bass, A. J., Watanabe, H., Mermel, C. H., Yu, S., Perner, S., Verhaak, R. G., et al. (2009). SOX2 is an amplified lineage-survival oncogene in lung and esophageal squamous cell carcinomas. Nat. Genet. 41, 1238-1242. doi: 10.1038/ng.465
(RIME) (Mohammed et al., 2016) or co-immunoprecipitation coupled with mass spectrometry for the identification of PPIs and the analysis of post-translational modifications. However, unbiased and high-throughput approaches to investigate interactions and post-translational modifications in a wholecell format are still lacking and therefore knowledge of particular TNBC oncoproteins is currently required to take this approach. The emerging field of single cell proteomics may offer the opportunity to perform unbiased screens to correlate particular protein states with cellular phenotypes in the future but, as of yet, high-throughput single-cell proteomics methods are not available for this purpose (Marx, 2019). However, the rate with which the single cell field is progressing bodes well for this technology and no doubt its development will offer unprecedented insight into PPIs driving malignancy. The combination of the above approaches may provide new therapeutic angles for the development of novel, more targeted and more effective TNBC therapies, as well as providing valuable insights into the mechanism of TNBC pathogenesis.

\section{AUTHOR CONTRIBUTIONS}

MW wrote the first draft of the manuscript and produced the figures. MW and RU wrote sections of the manuscript. All authors contributed to the manuscript revision, read, and approved the submitted version.

\section{FUNDING}

RU is funded by a NC3Rs studentship. MW is funded by a CRUK grant to WK. WK is funded by a CRUK Career Establishment Award (C47525/A17348), CRUK Small Molecule Drug Discovery Project Award (C47525/A25850), Breast Cancer Now Project Grant (2017MayPR907), and University of Cambridge and Magdalene College, Cambridge.

Bhola, N. E., Balko, J. M., Dugger, T. C., Kuba, M. G., Sánchez, V., Sanders, M., et al. (2013). TGF- $\beta$ inhibition enhances chemotherapy action against triple-negative breast cancer. J. Clin. Invest. 123, 1348-1358. doi: 10.1172/JCI6 5416

Bray, F., Ferlay, J., Soerjomataram, I., Siegel, R. L., Torre, L. A., and Jemal, A. (2018). Global cancer statistics 2018: GLOBOCAN estimates of incidence and mortality worldwide for 36 cancers in 185 countries. CA Cancer J. Clin. 68, 394-424. doi: 10.3322/caac.21492

Burstein, M. D., Tsimelzon, A., Poage, G. M., Covington, K. R., Contreras, A., Fuqua, S. A. W., et al. (2015). Comprehensive genomic analysis identifies novel subtypes and targets of triple-negative breast cancer. Clin. Cancer Res. 21, 1688-1698. doi: 10.1158/1078-0432.CCR-14-0432

Chen, L., Deshpande, A. J., Banka, D., Bernt, K. M., Dias, S., Buske, C., et al. (2013). Abrogation of MLL-AF10 and CALM-AF10-mediated transformation through genetic inactivation or pharmacological inhibition of the H3K79 methyltransferase Dot1l. Leukemia 27, 813-822. doi: 10.1038/leu.2012.327

Curtis, C., Shah, S. P., Chin, S. F., Turashvili, G., Rueda, O. M., Dunning, M. J., et al. (2012). The genomic and transcriptomic architecture of 2,000 breast tumours reveals novel subgroups. Nature 486, 346-352. doi: 10.1038/nature10983 
Daigle, S. R., Olhava, E. J., Therkelsen, C. A., Majer, C. R., Sneeringer, C. J., Song, J., et al. (2011). Selective killing of mixed lineage Leukemia cells by a potent smallmolecule DOT1L inhibitor. Cancer Cell 20, 53-65. doi: 10.1016/j.ccr.2011.06. 009

Dawson, S. J., Rueda, O. M., Aparicio, S., and Caldas, C. (2013). A new genomedriven integrated classification of breast cancer and its implications. EMBO J. 32, 617-628. doi: 10.1038/emboj.2013.19

Dent, R., Trudeau, M., Pritchard, K. I., Hanna, W. M., Kahn, H. K., Sawka, C. A., et al. (2007). Triple-negative breast cancer: clinical features and patterns of recurrence. Clin. Cancer Res. 13(15 Pt 1), 4429-4434. doi: 10.1158/1078-0432. CCR-06-3045

Deshaies, R. J., and Joazeiro, C. A. P. (2009). RING domain E3 ubiquitin ligases. Annu. Rev. Biochem. 78, 399-434. doi: 10.1146/annurev.biochem.78.101807. 093809

DiMeo, T. A., Anderson, K., Phadke, P., Feng, C., Perou, C. M., Naber, S., et al. (2009). A novel lung metastasis signature links Wnt signaling with cancer cell self-renewal and epithelial-mesenchymal transition in basal-like breast cancer. Cancer Res. 69, 5364-5373. doi: 10.1158/0008-5472.CAN-08-4135

Dolgin, E. (2019). Atezolizumab combo approved for PD-L1-positive TNBC. Cancer Discov. 9:OF2. doi: 10.1158/2159-8290.CD-NB2019-038

Engel, C., Rhiem, K., Hahnen, E., Loibl, S., Weber, K. E., Seiler, S., et al. (2018). Prevalence of pathogenic BRCA1/2 germline mutations among 802 women with unilateral triple-negative breast cancer without family cancer history. BMC Cancer 18:265. doi: 10.1186/s12885-018-4029-y

Fallahpour, S., Navaneelan, T., De, P., and Borgo, A. (2017). Breast cancer survival by molecular subtype: a population-based analysis of cancer registry data. CMAJ Open 5, E734-E739. doi: 10.9778/cmajo.20170030

Farmer, H., McCabe, H., Lord, C. J., Tutt, A. H. J., Johnson, D. A., Richardson, T. B., et al. (2005). Targeting the DNA repair defect in BRCA mutant cells as a therapeutic strategy. Nature 434, 917-921. doi: 10.1038/nature0 3445

Foulkes, W. D., Smith, I. E., and Reis-Filho, J. S. (2010). Triple-negative breast cancer. N. Engl. J. Med. 363, 1938-1948. doi: 10.1056/NEJMra1001389

Fowler, T., Ghatak, P., Price, D. H., Conaway, R., Conaway, J., Chiang, C. M., et al. (2014). Regulation of MYC expression and differential JQ1 sensitivity in cancer cells. PLoS One 9:e87003. doi: 10.1371/journal.pone.0087003

Fox, E. J. (2004). Mechanism of action of mitoxantrone. Neurology 63(12 Suppl. 6), S15-S18.

Franklin, M. C., Carey, K. D., Vajdos, F. F., Leahy, D. J., De Vos, A. M., and Sliwkowski, M. X. (2004). Insights into ErbB signaling from the structure of the ErbB2-pertuzumab complex. Cancer Cell 5, 317-328. doi: 10.1016/S15356108(04)00083-2

Fredly, H., Gjertsen, B. T., and Bruserud, Ø (2013). Histone deacetylase inhibition in the treatment of acute myeloid leukemia: the effects of valproic acid on leukemic cells, and the clinical and experimental evidence for combining valproic acid with other antileukemic agents. Clin. Epigenet. 5:12. doi: 10.1186/ 1868-7083-5-12

Gatalica, Z., Snyder, C., Maney, T., Ghazalpour, A., Holterman, D. A., Xiao, N., et al. (2014). Programmed cell death 1 (PD-1) and its ligand (PD-L1) in common cancers and their correlation with molecular cancer type. Cancer Epidemiol. Biomarkers Prev. 23, 2965-2970. doi: 10.1158/1055-9965.EPI-14-0654

Gewirtz, D. A. (1999). A critical evaluation of the mechanisms of action proposed for the antitumor effects of the anthracycline antibiotics adriamycin and daunorubicin. Biochem. Pharmacol. 57, 727-741. doi: 10.1016/S0006-2952(98) 00307-4

Goodsell, D. S. (1999). The molecular perspective: methotrexate. Oncology 4, 340-341.

Grandori, C., Cowley, S. M., James, L. P., and Eisenman, R. N. (2000). The $\mathrm{Myc} / \mathrm{Max} / \mathrm{Mad}$ network and the transcriptional control of cell behavior. Annu. Rev. Cell Dev. Biol. 16, 653-699. doi: 10.1146/annurev.cellbio.16.1.653

Griguolo, G., Dieci, M. V., Guarneri, V., and Conte, P. F. (2018). Olaparib for the treatment of breast cancer. Expert Rev. Anticancer Ther. 18, 519-530. doi: 10.1080/14737140.2018.1458613

Guenther, M. G., Jenner, R. G., Chevalier, B., Nakamura, T., Croce, C. M., Canaani, E., et al. (2005). Global and Hox-specific roles for the MLL1 methyltransferase. Proc. Natl. Acad. Sci. U.S.A. 102, 8603-8608. doi: 10.1073/pnas.050307 2102
Hall, A. G., and Tilby, M. J. (1992). Mechanisms of action of, and modes of resistance to, alkylating agents used in the treatment of haematological malignancies. Blood Rev. 6, 163-173. doi: 10.1016/0268-960X(92)90028-O

Harrison, H., Farnie, G., Howell, S. J., Rock, R. E., Stylianou, S., Brennan, K. R., et al. (2010). Regulation of breast cancer stem cell activity by signaling through the Notch4 receptor. Cancer Res. 70, 709-718. doi: 10.1158/0008-5472.CAN09-1681

Heale, J. T., Ball, A. R., Schmiesing, J. A., Kim, J. S., Kong, X., Zhou, S., et al. (2006). Condensin I interacts with the PARP-1-XRCC1 complex and functions in DNA single-strand break repair. Mol. Cell 21, 837-848. doi: 10.1016/j.molcel.2006.01. 036

Horwitz, S. B. (1994). Taxol (paclitaxel): mechanisms of action. Ann. Oncol. 5(Suppl. 6), S3-S6.

Junttila, T. T., Akita, R. W., Parsons, K., Fields, C., Lewis Phillips, G. D., Friedman, L. S., et al. (2009). Ligand-Independent HER2/HER3/PI3K complex is disrupted by trastuzumab and is effectively inhibited by the PI3K inhibitor GDC-0941. Cancer Cell 15, 429-440. doi: 10.1016/j.ccr.2009.03.020

Karatas, H., Li, Y., Liu, L., Ji, J., Lee, S., Chen, Y., et al. (2017). Discovery of a highly potent, cell-permeable macrocyclic peptidomimetic (MM-589) targeting the WD repeat domain 5 protein (WDR5)-mixed lineage leukemia (MLL) proteinprotein interaction. J. Med. Chem. 60, 4818-4839. doi: 10.1021/acs.jmedchem. 6b01796

Kawagoe, H., Humphries, R. K., Blair, A., Sutherland, H. J., and Hogge, D. D. (1999). Expression of HOX genes, HOX cofactors, and MLL in phenotypically and functionally defined subpopulations of leukemic and normal human hematopoietic cells. Leukemia 13, 687-698. doi: 10.1038/sj.leu.2401410

Koboldt, D. C., Fulton, R. S., McLellan, M. D., Schmidt, H., Kalicki-Veizer, J., McMichael, J. F., et al. (2012). Comprehensive molecular portraits of human breast tumours. Nature 490, 61-70. doi: 10.1038/nature11412

Konstantinopoulos, P. A., and Papavassiliou, A. G. (2011). Seeing the future of cancer-associated transcription factor drug targets. JAMA J. Am. Med. Assoc. 305, 2349-2350. doi: 10.1001/jama.2011.727

Krivtsov, A. V., Feng, Z., Lemieux, M., Faber, J., Xia, X., Kung, A. L., et al. (2007). Global increase in H3K79 dimethylation in murine and human MLL-AF4 lymphoblastic Leukemias. Blood 110:108A.

Le, D., and Gelmon, K. A. (2018). Olaparib tablets for the treatment of germ line BRCA-mutated metastatic breast cancer. Expert Rev. Clin. Pharmacol. 11, 833-839. doi: 10.1080/17512433.2018.1513321

Lehmann, B. D., Bauer, J. A., Chen, X., Sanders, M. E., Chakravarthy, A. B., Shyr, Y., et al. (2011). Identification of human triple-negative breast cancer subtypes and preclinical models for selection of targeted therapies. J. Clin. Invest. 121, 2750-2767. doi: 10.1172/JCI45014

Lehmann, B. D., Jovanović, B., Chen, X., Estrada, M. V., Johnson, K. N., Shyr, Y., et al. (2016). Refinement of triple-negative breast cancer molecular subtypes: implications for neoadjuvant chemotherapy selection. PLoS One 11:e0157368. doi: 10.1371/journal.pone.0157368

Levy, D. E., and Darnell, J. E. (2002). STATs: transcriptional control and biological impact. Nat. Rev. Mol. Cell Biol. 3, 651-662. doi: 10.1038/nrm909

Li, D. D., Chen, W. L., Wang, Z. H., Xie, Y. Y., Xu, X. L., Jiang, Z. Y., et al. (2016). High-affinity small molecular blockers of mixed lineage leukemia 1 (MLL1)WDR5 interaction inhibit MLL1 complex H3K4 methyltransferase activity. Eur. J. Med. Chem. 124, 480-489. doi: 10.1016/j.ejmech.2016.08.036

Li, W., Bengtson, M. H., Ulbrich, A., Matsuda, A., Reddy, V. A., Orth, A., et al. (2008). Genome-wide and functional annotation of human E3 ubiquitin ligases identifies MULAN, a mitochondrial E3 that regulates the organelle's dynamics and signaling. PLoS One 3:e1487. doi: 10.1371/journal.pone.0001487

Liu, S., Dontu, G., Mantle, I. D., Patel, S., Ahn, N. S., Jackson, K. W., et al. (2006). Hedgehog signaling and Bmi-1 regulate self-renewal of normal and malignant human mammary stem cells. Cancer Res. 66, 6063-6071. doi: 10.1158/00085472.CAN-06-0054

Lluís, F., Ballestar, E., Suelves, M., Esteller, M., and Muñoz-Cánoves, P. (2005). E47 phosphorylation by 38 MAPK promotes MyoD/E47 association and muscle-specific gene transcription. EMBO J. 24, 974-984. doi: 10.1038/sj.emboj. 7600528

Longley, D. B., Harkin, D. P., and Johnston, P. G. (2003). 5-Fluorouracil: mechanisms of action and clinical strategies. Nat. Rev. Cancer. 3, 330-338. doi: $10.1038 / \mathrm{nrc} 1074$ 
Lumachi, F., Brunello, A., Maruzzo, M., Basso, U., and Basso, S. (2013). Treatment of estrogen receptor-positive breast cancer. Curr. Med. Chem. 20, 596-604. doi: 10.2174/092986713804999303

Lüscher, B. (2001). Function and regulation of the transcription factors of the Myc/Max/Mad network. Gene 277, 1-14. doi: 10.1016/S0378-1119(01)00 697-7

Maier, H. J., Marienfeld, R., Wirth, T., and Baumann, B. (2003). Critical role of RelB serine 368 for dimerization and p100 stabilization. J. Biol. Chem. 278, 39242-39250. doi: 10.1074/jbc.M301521200

Marotta, L. L. C., Almendro, V., Marusyk, A., Shipitsin, M., Schemme, J., Walker, S. R., et al. (2011). The JAK2/STAT3 signaling pathway is required for growth of CD44 +CD24- stem cell-like breast cancer cells in human tumors. J. Clin. Invest. 121, 2723-2735. doi: 10.1172/JCI44745

Marx, V. (2019). A dream of single-cell proteomics. Nat. Methods 16, 809-812. doi: 10.1038/s41592-019-0540-6

Mathur, R., Alver, B. H., San Roman, A. K., Wilson, B. G., Wang, X., Agoston, A. T., et al. (2017). ARID1A loss impairs enhancer-mediated gene regulation and drives colon cancer in mice. Nat. Genet. 49, 296-302. doi: 10.1038/ng.3744

Mohammed, H., Taylor, C., Brown, G. D., Papachristou, E. K., Carroll, J. S., and D'Santos, C. S. (2016). Rapid immunoprecipitation mass spectrometry of endogenous proteins (RIME) for analysis of chromatin complexes. Nat. Protoc. 11, 316-326. doi: 10.1038/nprot.2016.020

Molina, M. A., Codony-Servat, J., Albanell, J., Rojo, F., Arribas, J., and Baselga, J. (2001). Trastuzumab (Herceptin), a humanized anti-HER2 receptor monoclonal antibody, inhibits basal and activated HER2 ectodomain cleavage in breast cancer cells. Cancer Res. 61, 4744-4749.

Morabito, F., Voso, M. T., Hohaus, S., Gentile, M., Vigna, E., Recchia, A. G., et al. (2016). Panobinostat for the treatment of acute myelogenous leukemia. Expert Opin. Investig. Drugs 25, 1117-1131. doi: 10.1080/13543784.2016.121 6971

Onitilo, A. A., Engel, J. M., Greenlee, R. T., and Mukesh, B. N. (2009). Breast cancer subtypes based on ER/PR and Her2 expression: comparison of clinicopathologic features and survival. Clin. Med. Res. 7, 4-13. doi: 10.3121/ cmr.2009.825

Partridge, A. H., Burstein, H. J., and Winer, E. P. (2001). Side effects of chemotherapy and combined chemohormonal therapy in women with earlystage breast cancer. J. Natl. Cancer Inst. Monogr. 2001, 135-142. doi: 10.1093/ oxfordjournals.jncimonographs.a003451

Peluffo, G., Subedee, A., Harper, N. W., Kingston, N., Jovanović, B., Flores, F., et al. (2019). EN1 is a transcriptional dependency in triple-negative breast cancer associated with brain metastasis. Cancer Res. 79, 4173-4183. doi: 10.1158/00085472.can-18-3264

Pierre, R. S., and Kadoch, C. (2017). Mammalian SWI/SNF complexes in cancer: emerging therapeutic opportunities. Curr. Opin. Genet. Dev. 42, 56-67. doi: 10.1016/j.gde.2017.02.004

Sakamoto, K. M., Kim, K. B., Kumagai, A., Mercurio, F., Crews, C. M., and Deshaies, R. J. (2001). Protacs: chimeric molecules that target proteins to the Skp1-Cullin-F box complex for ubiquitination and degradation. Proc. Natl. Acad. Sci. U.S.A. 98, 8554-8559. doi: 10.1073/pnas.141230798

Schmid, P., Adams, S., Rugo, H. S., Schneeweiss, A., Barrios, C. H., Iwata, H., et al. (2018). Atezolizumab and nab-paclitaxel in advanced triple-negative breast cancer. N. Engl. J. Med. 380, 987-988. doi: 10.1056/NEJMoa1809615

Schulman, B. A., and Wade Harper, J. (2009). Ubiquitin-like protein activation by E1 enzymes: the apex for downstream signalling pathways. Nat. Rev. Mol. Cell Biol. 10, 319-331. doi: 10.1038/nrm 2673
Shi, Y., Jin, J., Ji, W., and Guan, X. (2018). Therapeutic landscape in mutational triple negative breast cancer. Mol. Cancer 17:99. doi: 10.1186/s12943-0180850-9

Shou, Y., Martelli, M. L., Gabrea, A., Qi, Y., Brents, L. A., Roschke, A., et al. (2000). Diverse karyotypic abnormalities of the c-myc locus associated with c-myc dysregulation and tumor progression in multiple myeloma. Proc. Natl. Acad. Sci. U.S.A. 97, 228-233. doi: 10.1073/pnas.97.1.228

Slamon, D. J., Leyland-Jones, B., Shak, S., Fuchs, H., Paton, V., Bajamonde, A., et al. (2001). Use of chemotherapy plus a monoclonal antibody against her2 for metastatic breast cancer that overexpresses HER2. N. Engl. J. Med. 344, 783-792. doi: 10.1056/NEJM200103153441101

Sørlie, T., Perou, C. M., Tibshirani, R., Aas, T., Geisler, S., Johnsen, H., et al. (2001). Gene expression patterns of breast carcinomas distinguish tumor subclasses with clinical implications. Proc. Natl. Acad. Sci. U.S.A. 98, 10869-10874. doi: 10.1073/pnas.191367098

Sørlie, T., Tibshirani, R., Parker, J., Hastie, T., Marron, J. S., Nobel, A., et al. (2003). Repeated observation of breast tumor subtypes in independent gene expression data sets. Proc. Natl. Acad. Sci. U.S.A. 100, 8418-8423. doi: 10.1073/ pnas.0932692100

Tomasz, M. (1995). Mitomycin C: small, fast and deadly (but very selective). Chem. Biol. 2, 575-579. doi: 10.1016/1074-5521(95)90120-5

Venne, A. S., Kollipara, L., and Zahedi, R. P. (2014). The next level of complexity: crosstalk of posttranslational modifications. Proteomics 14, 513-524. doi: 10. 1002/pmic.201300344

Wahba, H. A., and El-Hadaad, H. A. (2015). Current approaches in treatment of triple-negative breast cancer. Cancer Biol. Med. 12, 106-116. doi: 10.7497/j.issn. 2095-3941.2015.0030

Wang, X., Lee, R. S., Alver, B. H., Haswell, J. R., Wang, S., Mieczkowski, J., et al. (2017). SMARCB1-mediated SWI/SNF complex function is essential for enhancer regulation. Nat. Genet. 49, 289-295. doi: 10.1038/ng.3746

Wertz, I. E., and Wang, X. (2019). From discovery to bedside: targeting the ubiquitin system. Cell Chem. Biol. 26, 156-177. doi: 10.1016/j.chembiol.2018. 10.022

Witwicki, R. M., Ekram, M. B., Qiu, X., Janiszewska, M., Shu, S., Kwon, M., et al. (2018). TRPS1 is a lineage-specific transcriptional dependency in breast cancer. Cell Rep. 25, 1255.e5-1267.e5. doi: 10.1016/j.celrep.2018.10.023

Woolfrey, K. M., and Dell'Acqua, M. L. (2015). Coordination of protein phosphorylation and dephosphorylation in synaptic plasticity. J. Biol. Chem. 290, 28604-28612. doi: 10.1074/jbc.R115.657262

Yan, C., and Higgins, P. J. (2013). Drugging the undruggable: transcription therapy for cancer. Biochim. Biophys. Acta Rev. Cancer 1835, 76-85. doi: 10.1016/j. bbcan.2012.11.002

Conflict of Interest: The authors declare that the research was conducted in the absence of any commercial or financial relationships that could be construed as a potential conflict of interest.

The handling Editor declared a past co-authorship with one of the authors WK.

Copyright (c) 2019 Waterhouse, Ugur and Khaled. This is an open-access article distributed under the terms of the Creative Commons Attribution License (CC BY). The use, distribution or reproduction in other forums is permitted, provided the original author(s) and the copyright owner(s) are credited and that the original publication in this journal is cited, in accordance with accepted academic practice. No use, distribution or reproduction is permitted which does not comply with these terms. 